\title{
Acute kidney injury electronic alerts: mixed methods evaluation of their implementation into secondary care, utilising normalisation process theory
}

\author{
Authors: Jason Scott, ${ }^{\mathrm{A}}$ Tracy Finch, ${ }^{\mathrm{A}}$ Gregory Maniatopoulos, ${ }^{\mathrm{B}}$ Mark Bevan ${ }^{\mathrm{A}}$ and Suren Kanagasundaram ${ }^{\mathrm{C}, \mathrm{D}}$
}

\begin{abstract}
Aims
With acute kidney injury (AKI) now a patient safety priority for NHS England, electronic AKI alerting systems have been mandated for all primary and secondary care providers under their aegis, using a biochemical detection algorithm incorporated into laboratory information management systems. The exact nature of the alerts is not dictated, and may be intrusive ('pop-ups'), passive (eg a notification within the biochemistry flowsheet), text message-based or supplemented by a human interaction by phone. Preliminary reports in general secondary care settings have yet to demonstrate improved clinical outcomes, and although poor implementation is linked to lack of success with similar technologies, few studies have specifically investigated the implementation of AKI alerts in depth. Our study therefore aimed to identify those factors which promoted or inhibited the use of AKI alerting systems in secondary care, using the evaluation framework of normalisation process theory (NPT).
\end{abstract}

\section{Methods}

Data were collected at three NHS trusts in north-east England, representing two distinct AKI e-alerting systems. Over 44 hours of observations were conducted in the emergency assessment unit (EAU) of each trust. Twenty-nine clinicians from EAUs, vascular and general surgery, and care of the elderly participated in semistructured interviews. Qualitative data were supplemented by an NPT-based questionnaire tool $(n=101$, representing a $65 \%$ response rate), which measures the normalisation of healthcare interventions. Qualitative data were analysed using the NPT framework, with quantitative data analysed descriptively and for differences in current and future normalisation.

\section{Results}

Participants reported familiarity with the e-alerts but felt that they would become more normalised in the future $(p<0.001)$.

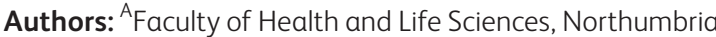
University; ${ }^{B}$ Institute of Health and Society, Newcastle University; ${ }^{C}$ Renal Services, Newcastle upon Tyne Hospitals NHS Foundation

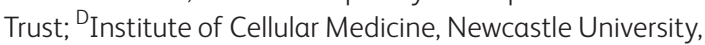
Newcastle upon Tyne, UK
Exploration of the four NPT mechanisms and their 16 underlying components identified no one NPT mechanism which affected the success of implementation alone. Analysis of the underlying components identified factors indicative of successful normalisation (understanding the potential benefit of the alerts, recognising the use of the alerts as being a legitimate part of their end-user role) and, conversely, unsuccessful normalisation (lack of training on the alerts, difficulty in recognising and prioritising AKI alerts, and lack of tangible impact data to learn from).

\section{Conclusion}

This is the first known mixed methods study to use NPT to investigate the implementation of AKI alerts. The findings suggest that while participants recognise the value of the AKI alerts in clinical practice, tailored training on how to use the alerts, and feedback to staff about their impact on outcomes, may promote improved staff engagement with AKI alerting systems.

\section{Conflict of interest statement}

None declared. 